\title{
Rehabilitation During a Pandemic: Psychiatrists as First Responders?
}

\author{
Jeremy Cave $\mathbb{B} \cdot$ Matthieu Crews
}

Received: 25 September 2020/Accepted: 1 October 2020/Published online: 22 October 2020

(C) Springer Nature India Private Limited 2020

\begin{abstract}
The South London and Maudsley Community Rehabilitation Psychiatry team aims to provide long-term and holistic care to patients with enduring mental illness. This letter concisely outlines our response to the coronavirus pandemic, including the standard operating procedure we introduced and, at a trust level, the changes made to clozapine monitoring. We were surprised by the expectations of our patients during the pandemic: we found that unwell patients or their carers would contact our service first, ahead of 111 , primary care or emergency services for advice and treatment. In response, we took on a deliberative first-responder role. Perhaps this is to be expected for a specialty that provides holistic long-term care to its patients. We think this is of interest to other mental health teams, primary care, community psychiatry teams and the lay reader.
\end{abstract}

Keywords Psychiatry - Rehabilitation - Clozapine · Primary care $\cdot$ Covid-19 $\cdot$ Holistic care

Community Rehabilitation Psychiatry aims to provide long-term, holistic, recovery focused care to patients

J. Cave $(\bowtie) \cdot$ M. Crews

South London and Maudsley NHS Foundation Trust, London, UK

e-mail: jeremy.cave@slam.nhs.uk with chronic mental-health conditions struggling with their social and personal functioning [3].

The South London and Maudsley High Support Rehabilitation Team serves 120 patients within the densely populated London borough of Southwark and responded to the challenges posed by Covid-19 at a team and trust level.

Generally our patients live in supported or residential placements, require higher-risk medication such as clozapine or augmented antipsychotic strategies and suffer poor physical health. Self-neglect and isolation are significant risks and a key part of our work is encouraging patients to attend primary care appointments and bringing together services in support of their recovery and inclusion.

For these reasons, Covid-19 poses a particular challenge to our cohort. Living in communal settings they are more likely to contract the virus and with multiple co-morbidities they are more likely to die if they become unwell. They require support to seek help, follow national guidelines and organise their medication and meals during isolation. For those on clozapine Covid-19 poses a diagnostic challenge with symptoms mimicking complications such as agranulocytosis and myocarditis. Routine white cell count monitoring and urgent blood counts for those unwell pose a transmission risk between staff and other patients.

Notably, we have found during the current pandemic that unwell patients or their carers would 
contact our service for advice ahead of 111, primary care or emergency services, perhaps reflecting the close trust they place in our team to support them through difficulty.

Anticipating the need to provide timely, evidencebased care, we devised and introduced same-day remote assessments structured around a standard operating procedure incorporating the latest primary care guidelines [2]. A doctor, often the core trainee, would assess the patient's physical and mental health, provide self-care and isolation advice and triage if required to further primary care (GP) input or the emergency services. Our care coordinators supported the patient with shopping and medication supplies for their isolation period and swab testing. We notified Public Health England if the criteria were met for a potential outbreak in a home. Recognising the possibility for patients to deteriorate around day 10 of their illness [2], all patients were reassessed the following week.

At a trust level, South London and Maudsley guidelines were amended permitting consultant discretion when deciding whether an urgent blood count was required for those unwell on clozapine [4] and routine blood count monitoring was extended to up to 3 months for eligible patients [1]. These changes acknowledge that some patients are at no greater risk of agranulocytosis than those on other antipsychotics [5] and frequent testing risks further community transmission. By requiring these changes, we suspect COVID-19 may have prompted a permanently less intensive monitoring regime for some patients on clozapine.

Reflecting on the role of rehabilitation psychiatry, the current pandemic has shown that community services are well placed to act as first responders to cases and, surprisingly, that this is expected of them by their patients. It is fitting, perhaps, for a service aiming to provide holistic care that its scope should have expanded in this way during the pandemic. That this primary care role is often performed by core trainees suggests a place for more rigorous primary care training within their Royal College curriculum.

Author Contributions Both authors have made substantial contributions to the design of the work. JC drafted the work and critically revised it, MC critically revised the work.

Funding This research received no specific grant from any funding agency, commercial or not-for-profit sector.

\section{Compliance with Ethical Standards}

Conflict of interest Both authors worked for the South London and Maudsley High Support Rehabilitation Team during the time of writing.

Consent for Publication Both authors approved this version for publication and both agree to be accountable for all aspects of the work.

\section{References}

1. Gee S, et al. Management of clozapine treatment during the COVID-19 pandemic. Ther Adv Psychopharmacol. 2020;10:2045125320928167. https://doi.org/10.1177/20451 25320928167.

2. Greenhalgh T, et al. Covid-19: a remote assessment in primary care. BMJ. 2020;368:m1182. https://doi.org/10.1136/ bmj.m1182.

3. Kalindindi S, Edwards T, Killaspy H. Community psychosis services: the role of community mental health rehabilitation teams. London: Royal College of Psychiatrists; 2012. 30 p. Report No: FR/RS/07.

4. Quality Centre of South London and Maudsley NHS Foundation Trust. SLAM Physical Health Guides 2020: Covid-19 infection in serious mental illness special considerations for outpatients on clozapine. London: South London and Maudsley NHS Foundation Trust. 2020 [updated 2020 May; cited 2020 Aug 20]

5. Schulte PF. Risk of clozapine-associated agranulocytosis and mandatory white blood cell monitoring. Ann Pharmacother. 2006;40:683-8. https://doi.org/10.1345/aph.1g396.

Publisher's Note Springer Nature remains neutral with regard to jurisdictional claims in published maps and institutional affiliations. 